\title{
Kinetics of Chitinase from Yam, Dioscorea opposita THUNB
}

\author{
Daizo Koga, * Tsuyoshi Tsukamoto, Nobuyuki Sueshige, \\ Toshihiko UTSUMI and Akio IDE \\ Laboratory of Biochemistry, Faculty of Agriculture, Yamaguchi University, \\ Yamaguchi 753, Japan \\ Received March 20, 1989
}

\begin{abstract}
Kinetic analysis was done on chitinase E3 (EC 3.2.1.14) from yam, Dioscorea opposita THUNB, using both series of $N$-acetylchitooligosaccharides ( $\operatorname{GlCNAc}_{n}, n=2$ to 6) and $p$-nitrophenyl $N$ acetylchitooligosaccharides (pNp-GlcNAc $c_{n}, n=1$ to 5) as substrates. The enzyme cleaved GlcNAc $c_{3}$ to GlcNAc plus GlcNAc ${ }_{2}, \mathrm{GlNNAc}_{4}$ to two molecules of GlcNAc ${ }_{2}, \mathrm{GlCNAc}_{5}$ to $\mathrm{GlcNAc}_{2}$ plus $\mathrm{GlcNAc}_{3}$, and $\mathrm{GleNAc}_{6}$ by three ways to GleNAc plus GleNAc $c_{5}(32 \%), \operatorname{GlNAc}_{2}$ plus GleNAc $\mathrm{C}_{4}(42 \%)$ and two molecules of $\mathrm{GlcNAc}_{3}(26 \%)$. The speed of the reaction was observed in the following order, GlcNAc$_{4}>$ GlcNAc $_{5}>\mathrm{GlcNAc}_{6}>\mathrm{GlcNAc}_{3}$. Stronger substrate inhibition was observed in the longer chain substrates. The reactions of $\mathrm{pNp}-\mathrm{GlCNAc}_{n}(n=1$ to 5$)$ were similar to those of $\operatorname{GlcNAc}_{n}(n=2$ to 6), respectively. The cleavage sites of pNp-GleNAc $c_{2}$ and pNp-GleNAc $c_{3}$ were the second $\beta$-1,4-linkages from the reducing end side, and the main cleavage sites of pNp-GIcNAc $c_{4}$ and pNp-GlcNAc $c_{5}$ were the third linkages. $p$-Nitrophenol was not released from all $p$-nitrophenyl derivatives. The speed of the reaction was observed in the following order, pNp-GleNAc ${ }_{4}>$ pNp-GleNAc $c_{5}>$ pNp-GlcNAc $>$ pNp$\mathrm{GlcNAc}_{2}$. Neither $\mathrm{GlCNAc}_{2}$ nor pNp-GlcNAc was cleaved. These results suggest that yam chitinase E3 acts in a random fashion.
\end{abstract}

Yam chitinase is good for releasing protoplasts from basidiomycetes, ${ }^{1)}$ and is resistant to allosamidin, which is a specific inhibitor of insect chitinase. ${ }^{2)}$ These characteristics are different from insect chitinases, and the differences seem to be related to their roles in physiology. In insects, two types of chitinolytic enzymes, chitinase and $\beta$ - $N$-acetylglucosaminidase, act cooperatively in ecdysis, ${ }^{3)}$ while the physiological role of plant chitinase is selfdefense against chitin-containing invaders such as plant pathogens and pests. ${ }^{4,5}$ It is easily expected that the reaction pattern and substrate specificity of yam chitinase are different from those of other chitinases.

In this study, we did a kinetic analysis on chitinase E3 from yam using both series of $N$-acetylchitooligosaccharides and $\mathrm{pNp}-N$ acetylchitooligosaccharides, and discussed the cleavage patterns and sites.

\section{Materials and Methods}

Materials. N-Acetylchitooligosaccharides (GlcNAc ${ }_{n}$, $n=1$ to 6) were prepared from crab chitin (a generous gift from Katakurachikkarin Co., Ltd., Tokyo) by the method of Raftery et al. ${ }^{6)} p$-Nitrophenyl $N$-acetylchitooligosaccharides (pNp-GlcNAc,$n=1$ to 5) were generous gifts from Yaizu Suisankagaku Industry Co., Ltd., Shizuoka, Japan. Other reagents were all of analytical grade.

Enzyme. Chitinase E3 was purified from yam, Dioscorea opposita THUNB, by the method of Tsukamoto et al. ${ }^{7)}$ and lyophilized for storage. The appropriate concentration of enzyme was prepared by dissolving the lyophilized enzyme in distilled water using a molar extinction coefficient of 47,000 . $^{7}$

Enzyme assay. Chitinase activity was measured by HPLC. Ten microliters of the enzyme solution (final concentration, 40 to $630 \mathrm{nM}$ ) was added to $100 \mu 1$ of $\operatorname{GleNAc}_{n}(n=2$ to 6) (final concentration, 0.05 to $0.2 \mathrm{~mm}$ ) or $\mathrm{pNp}-\mathrm{GicNAc}_{n}(n=1$ to 5) (final concentration, $0.1 \mathrm{~mm}$ )

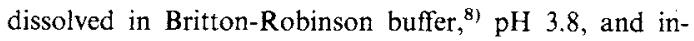

* To whom correspondence and reprint requests should be addressed.

Abbreviations: GlcNAc, 2-acetamido-2-deoxy- $\beta$-D-glucopyranoside; GlcNAc ${ }_{n}, \quad N$-acetylchitooligosaccharides; HPLC, high performance liquid chromatography; $\mathrm{pNp}-\mathrm{GlcNAc}_{n}, p$-nitrophenyl $N$-acetylchitooligosaccharides. 
cubated at $25^{\circ} \mathrm{C}$. After an appropriate time (10 to $\left.90 \mathrm{~min}\right)$, the reaction was stopped by boiling for $5 \mathrm{~min}$. A $10-\mu 1$ portion of the reaction mixture was injected into a Tosoh TSK Gel G2000 PW column $(0.75 \times 60 \mathrm{~cm})$ with Tosoh TSK PWL Guard column $(0.75 \times 7.5 \mathrm{~cm})$ as a first column, and eluted with distilled water or $10 \%$ ethanol, or injected into a Shimadzu $\mathrm{PNH}_{2}-10$ column $(0.45 \times 25 \mathrm{~cm})$ and eluted with $85 \%$ ethanol. The $N$-acetylchitooligosaccharides eluted were monitored at $210 \mathrm{~nm}^{9}{ }^{9}$

Kinetic analysis. Kinetic parameters were calculated by the non-linear optimization method described elsewhere ${ }^{10)}$ with a NEC ACOS-850 computer (Yamaguchi University).

\section{Results}

\section{Substrate specificity and cleavage pattern}

To investigate the substrate specificity and cleavage pattern, the enzymatic reactions were done with $\operatorname{GlcNAc}_{n}(n=2$ to 6$)$ as the substrates. The results are shown in Table I. Yam chitinase E3 cleaved $\mathrm{GlNAc}_{3}$ to GlcNAc plus $\mathrm{GlcNAc}_{2}, \mathrm{GlcNAc}_{4}$ to two molecules of $\mathrm{GlcNAc}_{2}, \mathrm{GlcNAc}_{5}$ to $\mathrm{GleNAc}_{2}$ plus $\mathrm{GlcNAc}_{3}$, and $\mathrm{GlcNAc}_{6}$ to all species of $\mathrm{GlcNAc}_{n}\left(n=1\right.$ to 5). GlcNAc$c_{2}$ was not cleaved. The release of GlcNAc was observed in the early reactions of $\mathrm{GlcNAc}_{3}$ and $\mathrm{GlcNAc}_{6}$. Products with longer chains than the substrate were not observed in any case. The speed of the reaction was observed in the following order, $\mathrm{GlcNAc}_{4}>\mathrm{GlcNAc}_{5}>\mathrm{GlcNAc}_{6}>$ $\mathrm{GlcNAc}_{3}$.

To locate the cleavage site, $\mathrm{pNp}$-GlcNAc $(n=1$ to 5$)$ were used as the substrates. The results are shown in Table II. The speed of the reaction was observed in the following order, pNp-GlcNAc $4>$ pNp-GlcNAc $>$ pNp-

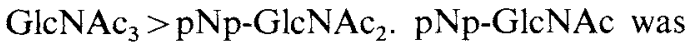
not cleaved. An endo-type cleavage pattern was observed except for pNp-GlcNAc ${ }_{2}$ which was cleaved very slowly to GlcNAc plus pNpGlcNAc. Release of $p$-nitrophenol was not observed in all cases. The cleavage sites of pNp-GlcNAc $c_{2}$ and pNp-GlcNAc 3 were the second $\beta$-1,4-linkages from the reducing end, and the main cleavage sites of $\mathrm{pNp}-\mathrm{GlcNAc}_{4}$ and $\mathrm{pNp}-\mathrm{GlcNAc}_{5}$ were the third linkages.

\section{Kinetic analysis}

We have conducted a detailed kinetic analysis of the hydrolysis of a series of $N$ acetylchitooligosaccharides ranging in chain length from 2 to 6 . The results are represented as double reciprocal plots in Figs. 1 and 2. Substrate inhibition was observed in the re-

Table I. Substrate Specificity of Yam Chitinase E3

Yam chitinase E3 ( $40 \mathrm{nM})$ was incubated with $0.1 \mathrm{~mm} \mathrm{~N}$-acetylchitooligosaccharide in Britton-Robinson buffer, $\mathrm{pH} 3.8$, at $25^{\circ} \mathrm{C}$. After an appropriate time, the reaction mixture was boiled for $5 \mathrm{~min}$, and a $10-\mu 1$ portion was analyzed by HPLC.

\begin{tabular}{|c|c|c|c|}
\hline Substrate & Reaction & $\begin{array}{c}\text { Initial velocity } \\
v_{0}(\mu \mathrm{m} / \mathrm{min})\end{array}$ & Cleavage pattern \\
\hline $\operatorname{GlcNAc}_{2}$ (II) & & 0 & and/or \\
\hline $\operatorname{GlcNAc}_{3}(\mathrm{III})$ & $\mathrm{III} \rightarrow \mathrm{I}+\mathrm{II}$ & $0.005 \pm 0.003$ & $\mathrm{G} \underline{G}_{\mathrm{G}} \unrhd_{\mathrm{G}}$ \\
\hline $\operatorname{GlcNAc}_{4}(\mathrm{IV})$ & IV $\rightarrow 2 \mathrm{II}$ & $1.782 \pm 0.067$ & $\begin{array}{c}\mathrm{G}-\mathrm{G}=\mathrm{G}-\mathrm{G} \\
\text { and/or }\end{array}$ \\
\hline $\mathrm{GlcNAc}_{5}(\mathrm{~V})$ & $\mathrm{V} \rightarrow \mathrm{II}+\mathrm{III}$ & $1.005 \pm 0.066$ & $\mathrm{G}-\mathrm{G} \unrhd_{\mathrm{G}} \unrhd_{\mathrm{G}-\mathrm{G}}$ \\
\hline \multirow[t]{4}{*}{$\mathrm{GlcNAc}_{6}(\mathrm{VI})$} & $\mathrm{VI} \rightarrow$ & $0.877 \pm 0.078(100 \%)$ & \\
\hline & $\rightarrow \mathrm{I}+\mathrm{V}$ & $0.281 \pm 0.011 \quad(32 \%)$ & $\begin{array}{c}\text { and/or } \\
G \unrhd G-G-G-G \unrhd_{G} \\
\text { and } / \text { or }\end{array}$ \\
\hline & $\rightarrow \mathrm{II}+\mathrm{IV}$ & $0.371 \pm 0.055 \quad(42 \%)$ & $\mathrm{G}-\mathrm{G}\rfloor_{\mathrm{G}-\mathrm{G}} \pm_{\mathrm{G}-\mathrm{G}}$ \\
\hline & $\rightarrow 2$ III & $0.225 \pm 0.017 \quad(26 \%)$ & $\mathrm{G}-\mathrm{G}-\mathrm{G} \downarrow \mathrm{G}-\mathrm{G}-\mathrm{G}$ \\
\hline
\end{tabular}

Mean values $\pm \mathrm{SE}, n=3$.

I, GlcNAc. 
Table II. Substrate Specificity of Yam Chitinase E3

The reactions were done as described in Table $\mathrm{I}$, but the substrates were $\mathrm{pNp}$ - $\mathrm{N}$-acetylchitooligosaccharides.

\begin{tabular}{|c|c|c|c|}
\hline Substrate & Reaction & $\begin{array}{l}\text { Initial velocity } \\
v_{0}(\mu \mathrm{m} / \mathrm{min})\end{array}$ & Cleavage pattern \\
\hline pNp-GicNAc (P-I) & & 0 & \\
\hline $\mathrm{pNp}-\mathrm{GlcNAc}_{2}(\mathrm{P}-\mathrm{II})$ & $\mathrm{P}-\mathrm{II} \rightarrow \mathrm{I}+\mathrm{P}-\mathrm{I}$ & $0.008 \pm 0.002$ & $\mathrm{G} \pm \mathrm{G}-\mathrm{P}$ \\
\hline $\begin{array}{l}\mathrm{pNp}-\mathrm{GlcNAc}_{3}(\mathrm{P}-\mathrm{III}) \\
\mathrm{pNp}-\mathrm{GlcNAc}_{4}(\mathrm{P}-\mathrm{IV})\end{array}$ & $\begin{array}{l}\mathrm{P}-\mathrm{III} \rightarrow \mathrm{II}+\mathrm{P}-\mathrm{I} \\
\mathrm{P}-\mathrm{IV} \rightarrow\end{array}$ & $\begin{array}{l}0.192 \pm 0.027 \\
1.499 \pm 0.057\end{array}$ & $G-G \perp G-P$ \\
\hline \multirow{5}{*}{$\mathrm{pNp}-\mathrm{GlcNAc} \mathrm{c}_{5}(\mathrm{P}-\mathrm{V})$} & $\rightarrow I I+P-I I$ & $1.172 \pm 0.046$ & $G-G \pm G-G-P$ \\
\hline & $\mathrm{P}-\mathrm{V} \rightarrow$ & $\begin{array}{l}0.327 \pm 0.062 \\
1.093 \pm 0.211\end{array}$ & $\mathrm{G}-\mathrm{G}-\mathrm{G}^{\mathfrak{d}} \mathrm{G}-\mathrm{P}$ \\
\hline & $\rightarrow \mathrm{II}+\mathrm{P}-\mathrm{III}$ & $0.277 \pm 0.064$ & G-G $\searrow_{\text {G-G-G-P }}$ \\
\hline & $\rightarrow \mathrm{III}+\mathrm{P}-\mathrm{II}$ & $0.619 \pm 0.156$ & $G-G-G \_G-G-P$ \\
\hline & $\rightarrow \mathrm{IV}+\mathrm{P}-\mathrm{I}$ & $0.157 \pm 0.036$ & G-G-G-G $\downarrow_{G-P}$ \\
\hline
\end{tabular}

Mean values $\pm \mathrm{SE}, n=3$.
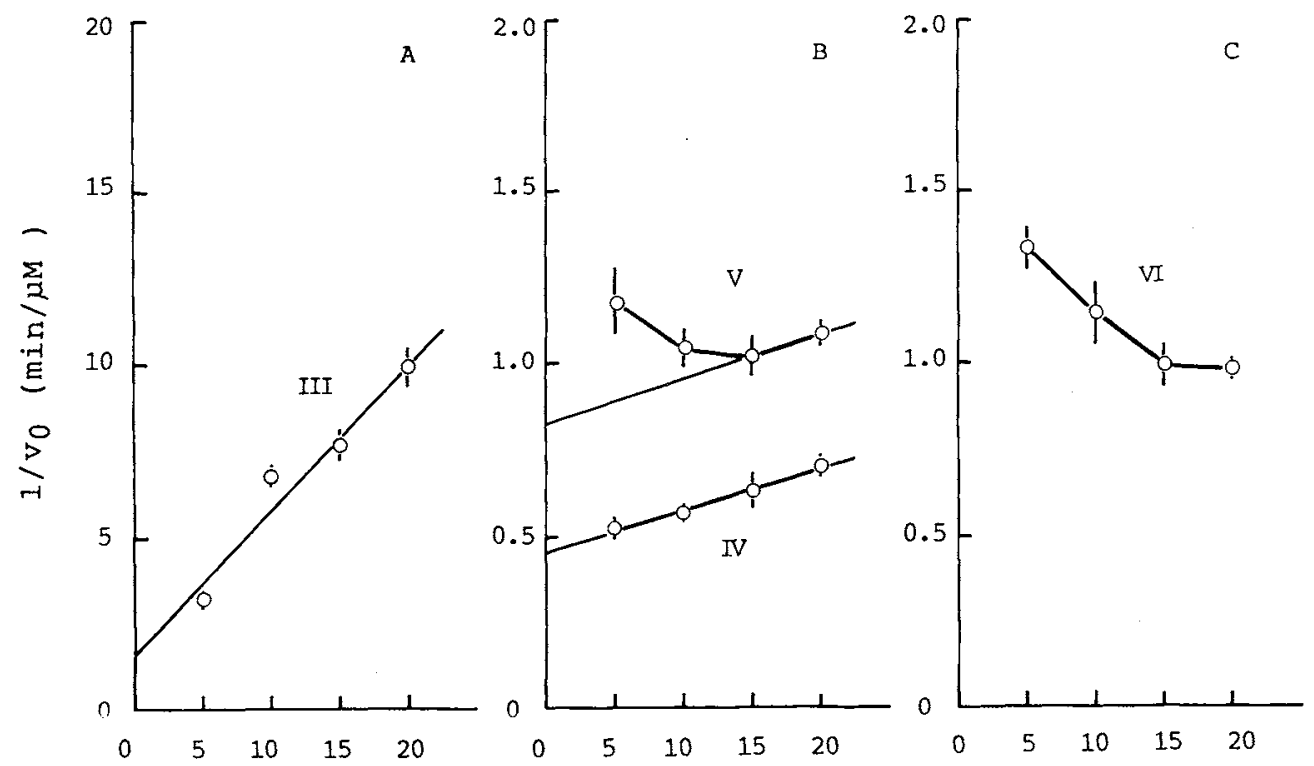

$$
1 / \mathrm{S}\left(\mathrm{mM}^{-1}\right)
$$

Fig. 1. Double Reciprocal Plots for Enzymatic Reactions of Yam Chitinase E3.

Ten microliters of the enzyme solution (final concentration, $40 \mathrm{nM}$ for GlcNAc $\mathrm{Gl}_{4}, \mathrm{GlNAc}_{5}$ and $\mathrm{GlcNAc}_{6}$ or $630 \mathrm{nM}$ for $\mathrm{GlcNAc}_{3}$ ) was added to $100 \mu \mathrm{l}$ of $\mathrm{N}$-acetylchitooligosaccharides (final concentrations, 0.05 to $0.2 \mathrm{~mm}$ ) dissolved in Britton-Robinson buffer, $\mathrm{pH} 3.8$, and incubated at $25^{\circ} \mathrm{C}$. After an appropriate time, the reaction mixture was boiled for $5 \mathrm{~min}$. A $10-\mu \mathrm{l}$ portion of the reaction mixture was analyzed by HPLC. See

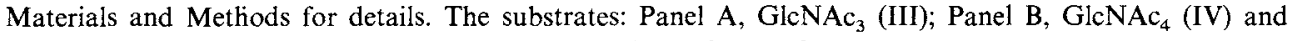
$\mathrm{GlcNAc}_{5}(\mathrm{~V})$; and Panel C, GlcNAc 6 (VI). Mean values $\pm \mathrm{SE}, n=3$. 


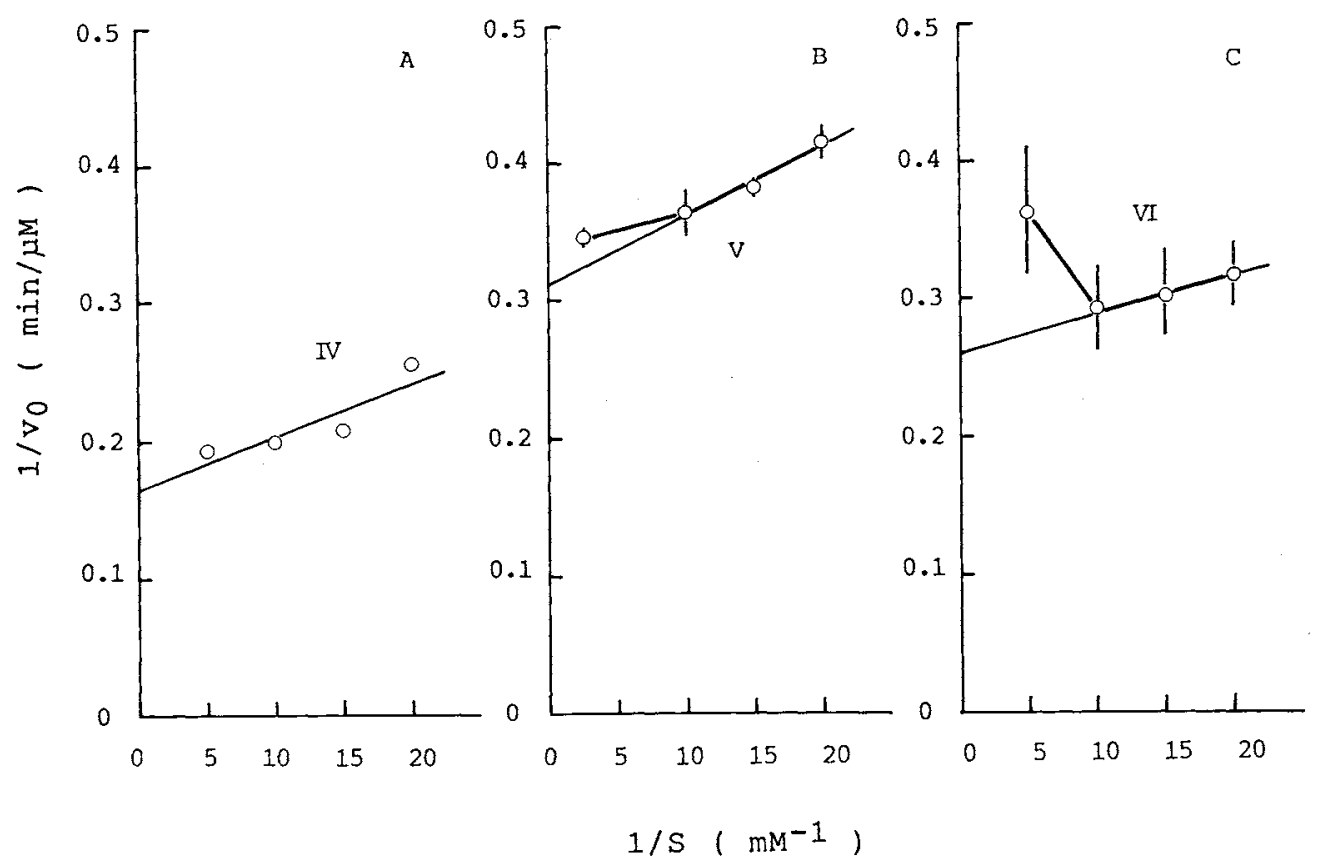

Fig. 2. Double Reciprocal Plots for Enzymatic Reactions of Yam Chitinase E3.

The enzymatic reactions and HPLC analysis were done as described in Fig. 1, but the final concentrations of the enzyme were $100 \mathrm{nM}$ for $\mathrm{GlcNAc}_{4}$ and $\mathrm{GlcNAc}_{5}$ and $160 \mathrm{~nm}$ for GlcNAc $\mathrm{c}_{6}$. The substrates: Panel A, $\mathrm{GlcNAc}_{4}$ (IV); Panel B, GlcNAc 5 (V); and Panel C, GlcNAc (VI). Mean values \pm SE, $n=3$ except for $\operatorname{GlcNAc}_{4}(n=1)$.

Table III. Kinetic Parameters for Yam Chitinase E3

The reactions were done as described in Table I. The kinetic parameters were calculated by the non-linear method. ${ }^{101}$

\begin{tabular}{|c|c|c|c|c|c|}
\hline \multirow{2}{*}{ Substrate } & \multicolumn{2}{|c|}{ Concentration } & \multirow{2}{*}{$k_{\text {cat }}\left(\sec ^{-1}\right)$} & \multirow{2}{*}{$K m(\mathrm{mM})$} & \multirow{2}{*}{$k_{\mathrm{cat}} / K m\left(\mathrm{mM}^{-1} \mathrm{sec}^{-1}\right)$} \\
\hline & Enz (nM) & $\operatorname{Sub}(\mu \mathrm{M})$ & & & \\
\hline $\mathrm{GlcNAc}_{3}$ & 630 & $50 \sim 200$ & $0.044 \pm 0.022$ & $0.880 \pm 0.527$ & 0.050 \\
\hline \multirow[t]{2}{*}{$\mathrm{GlcNAc}_{4}$} & 40 & $50 \sim 200$ & $0.913 \pm 0.049$ & $0.025 \pm 0.006$ & 36.2 \\
\hline & 100 & $50 \sim 200$ & $0.990 \pm 0.099$ & $0.020 \pm 0.010$ & 49.0 \\
\hline \multirow[t]{2}{*}{$\mathrm{GlcNAc}_{5}$} & 40 & 50,67 & $0.513 \pm 0.115$ & $0.014 \pm 0.017$ & 36.6 \\
\hline & 100 & $50 \sim 100$ & $0.540 \pm 0.040$ & $0.017 \pm 0.007$ & 32.4 \\
\hline $\mathrm{GlcNAc}_{6}$ & 160 & $50 \sim 100$ & $0.403 \pm 0.072$ & $0.010 \pm 0.014$ & 38.7 \\
\hline
\end{tabular}

Mean values \pm SE, $n=4 \sim 12$

actions of the longer chain substrates at the low concentration of the enzyme ( $40 \mathrm{~nm}$ ). However, it decreased or disappeared at the high concentration of the enzyme (100 or $160 \mathrm{~nm}$ ). The kinetic parameters calculated by the non-linear optimization method are shown in Table III. The $K m$ decreased as the substrate chain length increased, while the $k_{\text {cat }}$ was not dependent on the chain length. A high $k_{\text {cat }}$ was observed in $\mathrm{GlcNAc}_{4}$. The relationships between the overall reactions $\left(k_{\text {cat }} / K m\right)$ and the chain lengths reflected the initial velocities indicated in Table I. 


\section{Discussion}

From the results of enzymatic reactions, it was found that yam chitinase E3 cleaves both $\mathrm{GlcNAc}_{n}(n=3$ to 6$)$ and $\mathrm{pNp}-\mathrm{GlcNAc}_{n}(n=2$ to 5 ). The reaction products suggest that yam chitinase is a random-type hydrolytic enzyme. $\mathrm{GlcNAc}_{4}$ was cleaved in a specific way to two molecules of $\mathrm{GlcNAc}_{2}$. However, there are some cleavage ways in the reactions of other substrates as indicated in Table I. From the reaction products of $\mathrm{GlcNAc}_{6}$, it may be easily seen that the substrate is cleaved in three ways, namely to GlcNAc plus GlcNAc , GlcNAc $_{2}$ plus $\mathrm{GlcNAc}_{4}$ and two molecules of $\mathrm{GlcNAc}_{3}$. However, there are two further possible ways for the cleavage reaction producing two hetero-products such as GlcNAc and $_{n}$ and $\mathrm{GlcNAc}_{m}(n \neq m)$. This should be also considered in the cases of $\mathrm{GlcNAc}_{3}$ and $\mathrm{GlcNAc}_{5}$. The results of the reactions of $\mathrm{pNp}-\mathrm{GlNNAc}_{n}$ ( $n=1$ to 5) may be useful to find the cleavage site of $N$-acetylchitooligosaccharides. As shown in Table II, the cleavage of $\mathrm{pNp}$ $\mathrm{GlcNAc}_{2}$ occurred at a specific site, the second linkage from the reducing end. Therefore, it may be deduced that the cleavage site of $\mathrm{GlcNAc}_{3}$ is the second linkage from the reducing end. Comparing with the reaction pattern of $\mathrm{pNp}-\mathrm{GlcNAc}_{4}$, it may be deduced that the main cleavage site of $\mathrm{GlcNAc}_{5}$ is the third linkage from the reducing end. However, the difference in conformation between $p$-nitrophenyl and GlcNAc moieties should be taken into consideration. Since $p$-nitrophenol was not released in all cases, it is thought that the first $\beta$-1,4-linkage from the $p$-nitrophenyl moiety at the reducing end would not be cleaved. It may be deduced that the $p$-nitrophenyl moiety is not bound to the binding site adjacent to the reactive site, or that at least one GlcNAc moiety is required for binding to the binding site at the reducing end. In the case of $\mathrm{GlcNAc}_{6}, p$-nitrophenol or GlcNAc was not released from $\mathrm{pNp}^{-\mathrm{GlcNAc}} \mathrm{N}_{5}$ although GlcNAc was released in the reaction of Glc$\mathrm{NAc}_{6}$. It may be deduced that the GlcNAc released from $\mathrm{GlcNAc}_{6}$ is the moiety at the re- ducing end, and that the corresponding $p$ nitophenyl moiety of $\mathrm{pNp}-\mathrm{GlcNAc}_{5}$ is not cleaved for its steric hindrance. Two cleavage ways of $\mathrm{GlcNAc}_{6}$ to GlcNAc , plus GlcNAc may be possible as indicated in Table I, be-

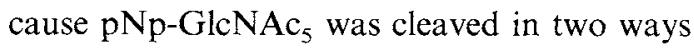
to $\mathrm{GlcNAc}_{2}$ plus pNp-GlcNAc and $_{3}$ alcNAc plus pNp-GlcNAc with the ratio of about $7: 4$. However, it is still difficult to decide which linkage is preferential, because the effect of $p$ nitrophenyl moiety on the binding is unknown. Comparing the cleaving rate of $\mathrm{GlcNAc}_{4}$ with that of pNp-GlcNAc ${ }_{3}$, it may be deduced that the $p$-nitrophenyl moiety strongly inhibited the reaction. It is suggested that the conformation is very crucial for the substrate to be recognized and bound to the binding sites adjacent to the reactive site. Therefore, some care is needed to decide the cleavage sites of $\mathrm{N}$-acetylchitooligosaccharides from the results of the reactions of $p$-nitrophenyl $N$-acetylchitooligosaccharides. The cleavage sites estimated as mentioned above are shown in Tables I and II, and the main cleavage sites are indicated by big arrows.

With respect to the substrate specificity, a faster reaction was observed in $\mathrm{GlcNAc}_{4}$ rather than GlcNAc 5 or GlcNAc 6 (Table I). The reaction velocity may be due to the probability of formation of the productive enzymesubstrate complex or to the degree of the affinity. However, as the substrate chain length increased from 3 to 6 , the $\mathrm{Km}$ decreased and the affinity increased. (Table III) That is, although the binding is strong, the reaction is slow. It may be deduced that the nonproductive enzyme-substrate complexs are more frequently formed in the cases of $\mathrm{GlcNAc}_{5}$ and $\mathrm{GlcNAc}_{6}$ than GlcNAc . $_{4}$

Comparing with insect chitinases from Manduca sexta ${ }^{11)}$ and Bombyx mori (Koga et al., unpublished data), the insect chitinases preferentially cleave $\mathrm{GlcNAc}_{5}$ and $\mathrm{GlcNAc}_{6}$ rather than $\mathrm{GlCNAc}_{4}$. The activities of the insect chitinases are about 4 times higher than that of yam chitinase toward $\mathrm{GlcNAc}_{5}$, and 2 or 3 times higher toward glycol chitin. ${ }^{7)}$ That 
is, the insect chitinases have higher activities than yam chitinase. However, the difference in the ratio between the long and short substrates seems to be due to the reactivity toward glycol chitin which is a chitin partially modified with ethylene glycol groups. The insect chitinases are largely affected by the degree of ethylene glycol groups, compared with yam chitinase. There is also a difference in the cleavage pattern of $\mathrm{GlcNAc}_{6}$ between yam chitinase and the insect enzymes. The insect chitinases cleave $\mathrm{GlcNAc}_{6}$ by only two ways to

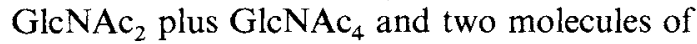
$\mathrm{GlcNAc}_{3}$, while yam chitinase does by three ways to GlcNAc plus GlcNAc $c_{5}$ in addition to the above two ways. (Table I) These differences in the enzymatic reaction between yam chitinase and the insect chitinases seem to be related to those in molecular size. The molecular weight of yam chitinase is $33.5 \mathrm{kDa}$, ${ }^{7)}$ while those of the insect chitinases are about 50 to $85 \mathrm{kDa} .{ }^{11,12)}$ The different characteristics in enzymatic reaction may be due to their physiological roles. Yam chitinase acts in selfdefense against plant pathogens (Koga et al., unpublished data) like other plant chitinases ${ }^{4,5)}$ while insect chitinase acts in ecdysis. ${ }^{3)}$ Therefore, plant chitinase may have a wide substrate specificity, while insect chitinase may have a strict one with high activity.

\section{References}

1) D. Koga, N. Sueshige, K. Orikono, T. Utsumi and A. Ide, Agric. Biol. Chem., 52, 2091 (1988).

2) D. Koga, A. Isogai, S. Sakuda, S. Matsumoto, A. Suzuki, S. Kimura and A. Ide, Agric. Biol. Chem., 51, 471 (1987).

3) K. J. Kramer and D. Koga, Insect Biochem., 16, 851 (1986).

4) A. Schlumbaum, F. Mauch, U. Vogeli and T. Boller, Nature, 324, 365 (1986).

5) R. Vogeli-Lange, A. Hansen-Gehri, T. Boller and F. Meins, Jr., Plant Science, 54, 171 (1988).

6) M. A. Raftery, T. Rand-Meir, F. W. Dahlquist, S. M. Parsons, C. L. Borders, R. G. Wolcott, W. Beraneck and L. Jao, Anal. Biochem., 30, 427 (1969).

7) T. Tsukamoto, D. Koga, A. Ide, T. Ishibashi, M. Horino-Matsushige, K. Yagishita and T. Imoto, Agric. Biol. Chem., 48, 931 (1984).

8) H. T. S. Britton and R. A. Robinson, J. Chem. Soc., $1931,1456$.

9) D. Koga, M. Nakashima, T. Matsukura, S. Kimura and A. Ide, Agric. Biol. Chem., 50, 2357 (1986).

10) D. Koga, M. S. Mai, C. Dziadik-Turner and K. J. Kramer, Insect Biochem., 12, 493 (1982).

11) D. Koga, J. Jilka and K. J. Kramer, Insect Biochem., 13, 295 (1983).

12) D. Koga, H. Fujimoto, T. Funakoshi, T. Utsumi and A. Ide, Insect Biochem., 19, 123 (1989). 